\title{
Contrast enhanced ultrasound (CEUS) to assess pleural pulmonal changes in severe COVID-19 infection: First results
}

\author{
E.M. Jung ${ }^{\mathrm{a}, *}$, C. Stroszczynski ${ }^{\mathrm{a}}$ and F. Jung ${ }^{\mathrm{b}}$ \\ ${ }^{a}$ Department of Radiology, University Medical Center, Regensburg, Germany \\ ${ }^{\mathrm{b}}$ Institute of Biotechnology, Brandenburg University of Technology, Senftenberg, Germany
}

\begin{abstract}
.
AIM: Use of contrast enhanced ultrasound (CEUS) in severe cases of COVID-19 infection to assess pulmonary changes near the pleura.

MATERIAL AND METHODS: Bedside examinations by an experienced intensive care unit examiner using a multifrequency probe (C1-6 MHz) with B-mode and CEUS to assess pleural-near changes in severe cases of COVID-19 infection with respiratory failure. CEUS with bolus delivery via a central venous catheter of $2.4 \mathrm{ml}$ Sulphur hexafluoride microbubbles from the arterial phase (10-15 s) to the late phase of $5 \mathrm{~min}$. Digital storage of cine sequences of the lung sound with abdomen for independent assessment with the subsequently performed contrast-enhanced dual-source CT.

RESULTS: In 11 intubated and ventilated patients (arithmetic mean 62 years, 48 to 78 years, 3 women) with confirmed severe COVID-19 infections, a peripherally accentuated consolidation with irregular hyperemia was found in the CEUS and also in the CT examination. Of the 5 cases with pulmonary arterial embolisms, signs of right ventricular failure were found. In all cases, using CEUS low perfused areas of the pleura with adjacent hyperemia could be detected, while, with CT segmental contrast medium, gaps with subpleural compressions were found. Interstitial changes near the pleura led to B-lines and to ground glass opacities in the CT. Near the diaphragm a delayed arterial contrast of the liver was observed. In addition, in 2 cases partial atelectasis, in 3 cases marginal pleural effusions were found.

CONCLUSION: CEUS opens up new possibilities for bedside monitoring of pleural reactive inflammatory or peripheral
\end{abstract} thrombus embolism in severe cases of COVID-19 infection.

Keywords: COVID-19 infection, lung- Contrast-enhanced ultrasound (CEUS), pleural affection, peripheral embolic consolidation

\section{Introduction}

In the course of the worldwide pandemic caused by COVID-19 infections, imaging of pulmonary changes is of great diagnostic importance. Early peripherally prominent reactive changes, mostly interstitial, but also severe courses with consolidations up to acute respiratory distress syndrome (ARDS) have to be assessed. Increasingly, both computed tomography (CT) and ultrasound diagnostics are used [1-3].

In case of indications of pulmonary arterial embolism (LAE), a spiral CT with contrast medium is performed. Not only central embolisms but also peripheral embolisms resulting in infarct pneumonia must be detected. This can be done safely up to segmental level. Further peripherally, the examination

\footnotetext{
${ }^{*}$ Corresponding author: Prof Dr med Ernst Michael Jung, Department of Radiology, University Medical Center, Regensburg, Germany. E-mail: ernst-michael.jung@ukr.de.
} 
conditions and the possibility to examine without respiratory artifacts are crucial for CT. This is where targeted ultrasound diagnostics can be used. It is important to differentiate between reactive inflammatory changes close to the pleura and post-embolic reduced ventilation. Without contrast medium, however, perfusion changes cannot be detected [2-7].

CEUS allows dynamically to detect perfusion changes down to capillary level using sulphur hexafluoride microbubbles as contrast medium (SonoVue/Bracco) [8, 9]. In addition, changes in organ perfusion in severe cases of COVID-19 infection in intubated and ventilated patients with impaired renal function can be recorded dynamically with CEUS [20]. While ultrasound examinations of the lung are increasingly used, CEUS for pulmonary changes is already part of the EFSUMB Guidelines, but is reserved for specific topics [10,11].

Aim of this pilot study was to detect pulmonary microcirculatory changes in severe cases of COVID19 infection using bedside CEUS diagnostics in intensive care units and to evaluate these changes in correlation to the independently performed CT.

\section{Material and methods}

For the assessment of pulmonary changes in COVID-19 infections, ethics and recommendations are available from the professional societies, which have been joined by local commissions in the context of the pandemic. In most cases, examinations are performed with B-mode sonography or native spiral CT [1, 5, 12-15].

In the clinical diagnosis of a suspected pulmonary arterial embolism the contrast medium enhanced spiral CT was used [5, 6, 14-18]. For this purpose, triggering was performed on the pulmonary trunk in order to achieve an early high contrast with 60 to $80 \mathrm{ml}$ iodine-containing contrast medium with the highest resolution by dual-source multislice CT (Somaton/Senation Plus/Siemens, Erlangen Germany) with reconstructions of slice thicknesses down to only $1 \mathrm{~mm}$ axial and coronary reconstructions.

COVID-19 infectious lesions were evaluated by 2 independent radiologists according to the expert recommendations regarding peripherally accentuated ground glass alterations as sign of early pulmonary changes or consolidations in advanced course. Thromboembolism was assessed as contrast gaps in the pulmonary arteries down to at least segmental level and accompanying peripheral blood flow loss.

After 2 to 5 days in the intensive care unit in severe cases of COVID 19 infection with respiratory obligation bedside examinations were performed by an experienced examiner using B-mode and CEUS to assess pleural changes and organ perfusion near the diaphragm. The examinations were carried out with a multi-frequency probe (C1-6 MHz) with a medium or high-end device (LOGIQ S8/ LOGIQ E9/GE), taking into account the special hygiene measures required.

CEUS was performed after bolus administration of $2.4 \mathrm{ml}$ sulphur hexafluoride microbubbles (SonoVue/BRACCO) with $10 \mathrm{ml}$ saline solution from arterial phase (10-15 s) to late phase of $5 \mathrm{~min}$. DICOM cine sequences of the lung with abdomen were digitally stored in the PACS for independent evaluation. By independent readers CEUS images of the clips were analysed. Contrast enhancement was documented and differentiated between inhomogeneous and absent enhancement. The inhomogeneous patchy pattern was characterized as vascularized lesions with coexisting pleural-shaped avascular areas of various extent considering the EFSUM Guidelines [11].

\section{Results}

Table 1 shows demographic and clinical data from patients included. 
Table 1

Demographic and clinical data

\begin{tabular}{cccccccccc}
\hline Age & Sex & $\begin{array}{c}\text { COVID-19 } \\
\text { infection, } \\
\text { respiratory } \\
\text { failure }\end{array}$ & $\begin{array}{c}\text { CT } \\
\text { ground } \\
\text { glas } \\
\text { opacity }\end{array}$ & $\begin{array}{c}\text { US/ } \\
\text { CEUS } \\
\text { B- }\end{array}$ & $\begin{array}{c}\text { CT/US } \\
\text { CEUS } \\
\text { consoli } \\
\text { dations }\end{array}$ & $\begin{array}{c}\text { CEUS } \\
\text { pleural } \\
\text { hyperemia }\end{array}$ & $\begin{array}{c}\text { CT } \\
\text { pulmonary } \\
\text { arterial } \\
\text { embolism }\end{array}$ & $\begin{array}{c}\text { CEUS } \\
\text { pleural } \\
\text { perfusion } \\
\text { defects }\end{array}$ & $\begin{array}{c}\text { CT/US } \\
\text { atelectasis }\end{array}$ \\
\hline 78 & $\mathrm{~m}$ & $\mathrm{X}$ & $\mathrm{X}$ & $\mathrm{X}$ & $\mathrm{X}$ & $\mathrm{X}$ & & \\
52 & $\mathrm{~m}$ & $\mathrm{X}$ & $\mathrm{X}$ & $\mathrm{X}$ & $\mathrm{X}$ & $\mathrm{X}$ & & $\mathrm{X}$ \\
55 & $\mathrm{~m}$ & $\mathrm{X}$ & $\mathrm{X}$ & $\mathrm{X}$ & $\mathrm{X}$ & $\mathrm{X}$ & $\mathrm{X}$ & $\mathrm{X}$ \\
66 & $\mathrm{~m}$ & $\mathrm{X}$ & $\mathrm{X}$ & $\mathrm{X}$ & $\mathrm{X}$ & $\mathrm{X}$ & $\mathrm{X}$ & \\
68 & $\mathrm{f}$ & $\mathrm{X}$ & $\mathrm{X}$ & $\mathrm{X}$ & $\mathrm{X}$ & $\mathrm{X}$ & & \\
61 & $\mathrm{~m}$ & $\mathrm{X}$ & $\mathrm{X}$ & $\mathrm{X}$ & $\mathrm{X}$ & $\mathrm{X}$ & $\mathrm{X}$ & $\mathrm{X}$ & \\
64 & $\mathrm{~m}$ & $\mathrm{X}$ & $\mathrm{X}$ & $\mathrm{X}$ & $\mathrm{X}$ & $\mathrm{X}$ & & $\mathrm{X}$ \\
65 & $\mathrm{f}$ & $\mathrm{X}$ & $\mathrm{X}$ & $\mathrm{X}$ & $\mathrm{X}$ & $\mathrm{X}$ & & $\mathrm{X}$ \\
55 & $\mathrm{~m}$ & $\mathrm{X}$ & $\mathrm{X}$ & $\mathrm{X}$ & $\mathrm{X}$ & $\mathrm{X}$ & $\mathrm{X}$ & $\mathrm{X}$ \\
68 & $\mathrm{~m}$ & $\mathrm{X}$ & $\mathrm{X}$ & $\mathrm{X}$ & $\mathrm{X}$ & $\mathrm{X}$ & $\mathrm{X}$ & $\mathrm{X}$ \\
48 & $\mathrm{f}$ & $\mathrm{X}$ & $\mathrm{X}$ & $\mathrm{X}$ & $\mathrm{X}$ & $\mathrm{X}$ & $\mathrm{X}$ & $\mathrm{X}$ \\
\hline
\end{tabular}
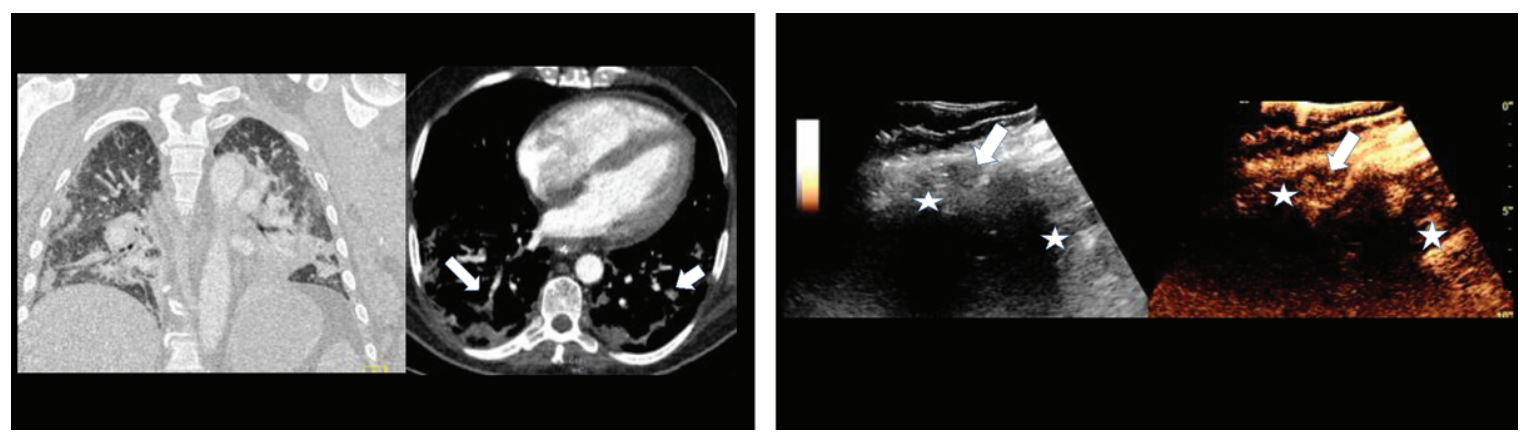

Fig. 1. 1A: Contrast enhanced computed tomography (CT) of a 48 years old male COVID-19 patient with pulmonary arterial embolism of the right lung (arrow) and consolidation on both sides. Documentation with two different windows for vascular structures and lung parenchymal changes.

In 11 intubated and ventilated patients (48 to 78 years, mean $61.8 \pm 8.7$ years, 3 women) with confirmed COVID-19 infections with severe course, a peripherally pronounced consolidation with irregular hyperemia were found in the CEUS and the CT examination. In addition, in two cases partial atelectasis, in three cases marginal angulation were detected. In the five patients with pulmonary embolism signs of right ventricular failure were found.

Figure 1: B-mode and contrast enhanced ultrasound (CEUS) of the same 48 years old male COVID19 patient with pulmonary arterial embolism of the right lung (arrow) and appositional consolidation. Embolism defects with irregular pleural effusions, central devascularization and marginal hyperenhancement. Irregular parenchymal hyperemia corresponding to the inflammatory consolidations (asterisk).

Lung ultrasound sign of inflammatory reactions include various forms of B-lines, irregular or fragmental pleural lines, pleural effusion and absence of lung sliding. Appositional an explosion of multiform vertical artefacts and separate and coalescent B-lines were detected. In 2 cases of ARDS the pleural lines were irregular or fragmented. In cases of pneumonia light beam artefact was observed. 

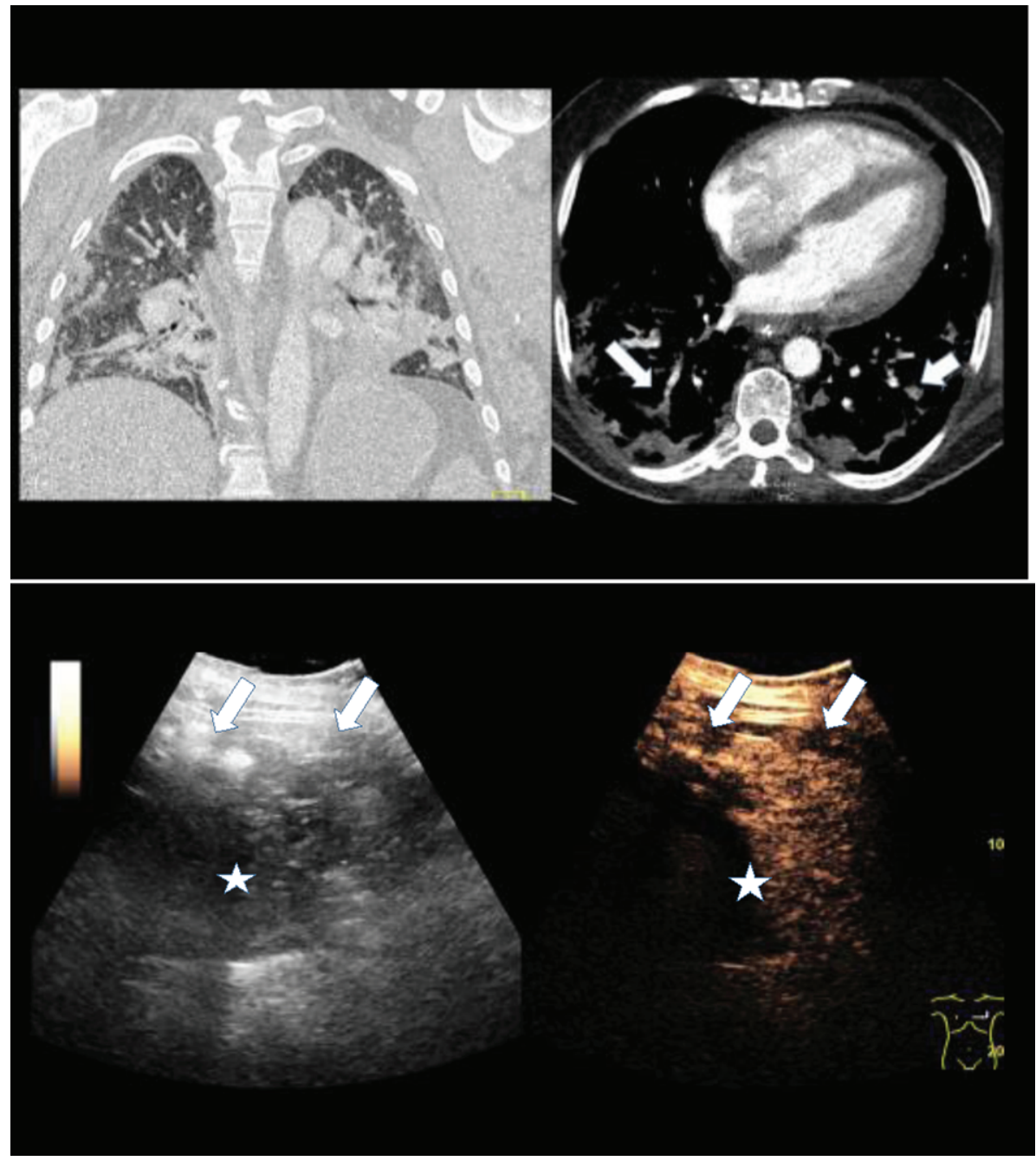

Fig. 2. 2A: Contrast enhanced computed tomography (CT) of a 56 years old female COVID-19 patient with pulmonary arterial embolism of the right lung (arrow) and consolidation on both sides. Documentation with two different windows for vascular structures and lung parenchymal changes.

This artefact corresponds to the early appearance of ground glass opacities detected in computed tomography.

Figure 2: B-mode and contrast enhanced ultrasound (CEUS) of the same 56 years old female COVID-19 patient with pulmonary arterial embolism (arrow) and appositional consolidation. Embolism defects with irregular pleural effusion, central devascularization and marginal hyperenhancement. Irregular parenchymal hyperemia corresponding to the inflammatory consolidations (asteriks).

In all 11 patients bilateral, patchy distribution of multiple cluster areas with the light beam sign, alternating with areas with multiple separated and coalescent B-lines and well-demarcated separation from large spared areas were observed. The pleural lines were partially irregular and fragmented. Sliding was preserved, multiple consolidations limited to the periphery of the lung and light beam artefacts could be visualized below small peripheral consolidations and zones with irregular pleural lines. Five patients with pulmonary arterial embolisms showed signs 
A

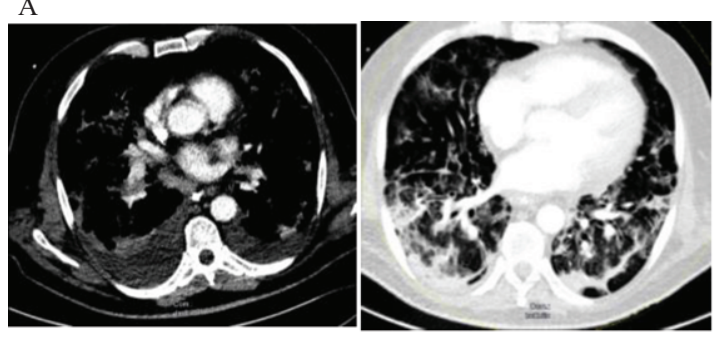

$\mathrm{C}$

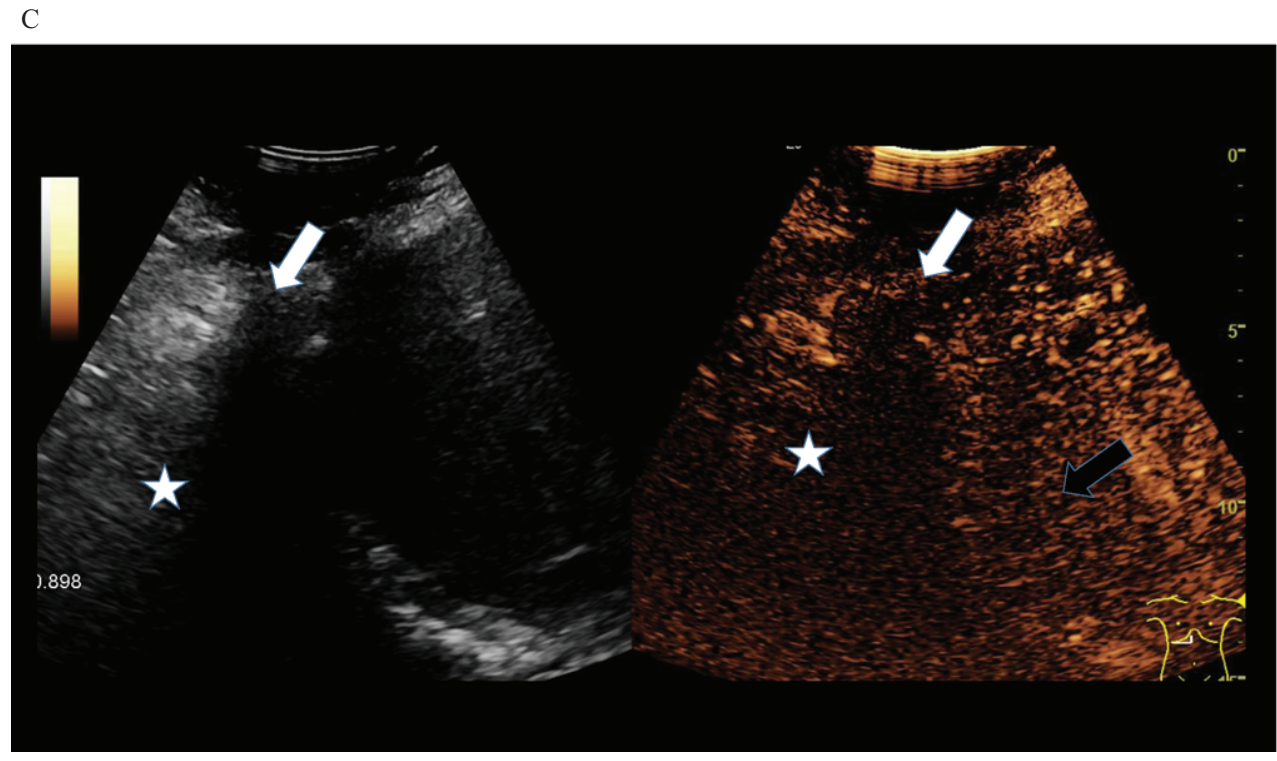

Fig. 3. 3A: Contrast enhanced computed tomography (CT) of a 55 year old female COVID-19 patient with pulmonary arterial embolism of the lung (arrow) and consolidation on both sides. Documentation with two different windows for vascular structures and lung parenchymal changes.

of right ventricular failure. In all cases of peripheral embolic lesions, using CEUS subpleural circumscripted centrally non-perfused areas of the pleura with adjacent marginal hyperemia could be detected, while with CT segmental lower contrasted areas with pleural effusion were found.

CEUS patterns of peripheral pleural lesions in the patients with confirmed pulmonary embolism were no enhancement and/or inhomogeneous enhancement with areas of no enhancement typically for peripheral embolism and more homogeneous arterial enhancement as a sign for inflammatory reactions. Pleural effusions detected by $\mathrm{CT}$ were associated with pulmonal arterial enhancement in CEUS corresponding to typical perfusion pattern of atelectatic consolidation. Lesions smaller than $10 \mathrm{~mm}$ in size showed no enhancement in the centre.

Figure 3: B-mode and contrast enhanced ultrasound (CEUS) of a 55 old female patient with pulmonary arterial embolism (arrow) and appositional consolidation. Embolism defects with irregular pleural enhancement, central devascularization, marginal hyperenhancement and pleural effusion. Irregular parenchymal enhancement and B-lines of inflammatory consolidation (asterisk). Reduced peripheral enhancement of the liver (black arrow).

Near the diaphragm a delayed arterial contrast of the liver near the capsule was observed in all cases. In 2 cases also perihepatic serous fluid was found. 

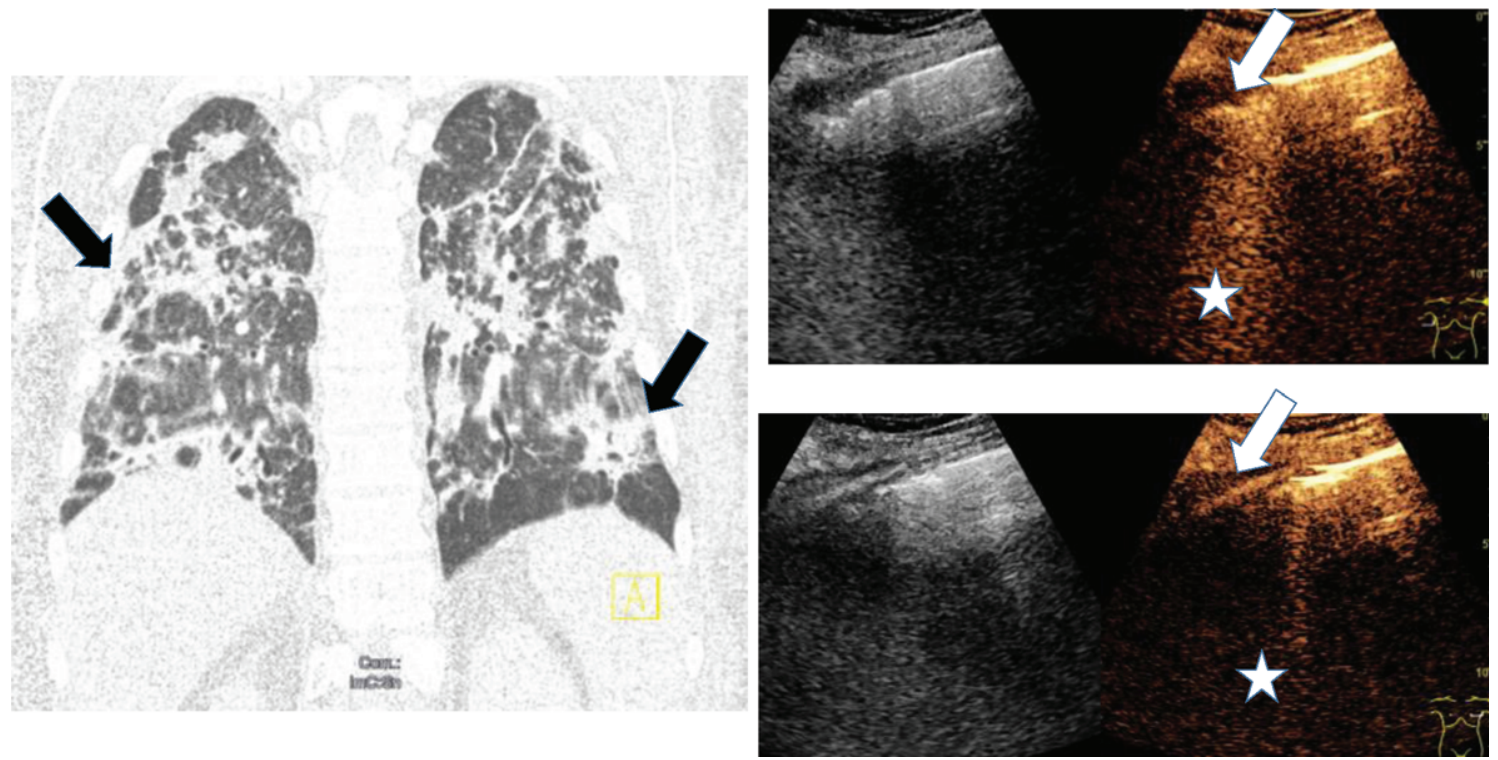

Fig. 4. Computed tomography (CT), B-mode and contrast enhanced ultrasound (CEUS) of a 68 old female COVID-19 patient with pulmonary infection (arrow) and pleural effusion. Irregular pleural enhancement, irregular parenchymal enhancement and B-lines of inflammatory consolidation (asterisk).

\section{Discussion}

The first results of this pilot study confirm the assessment that by means of contrast-enhanced ultrasound (CEUS) peripheral blood flow deficiencies, embolisms in the context of pulmonary artery embolism, microinfarcts and reactive hyperemia in the case of consolidation and pleural irritation in the pulmonary periphery can also be detected bedside. Thus, CEUS can contribute to an improvement in the follow-up of severe infection constellations and embolisms in COVID-19 infections.

The signs seen in the B-mode ultrasound of patients with COVID-19 are similar to those extensively described in patients with other types of pneumonia. These include various forms of B-lines, an irregular or fragmented pleural line, consolidations, pleural effusions and absence of lung sliding. The lung ultrasound (LUS) of patients with COVID-19 usually shows an explosion of multiform vertical artifacts and separate and coalescent B-lines. The pleural line may be irregular or fragmented as is commonly observed in ARDS. As stated above none of these signs is pathognomonic to COVID-19 pneumonia and their presence is variable [1].

The fact that CEUS has a high diagnostic potential for the detection of peripheral embolisms and reduced blood flow in the lung was already mentioned in the recommendations of the EFSUMB guidelines and the results of other working groups $[11,17,18]$. In COVID-19, CEUS is limited in severe disease progression by the fact that the use of sulphur hexafluoride microbubbles can lead to right heart failure with pulmonary hypertension. Often, restricted right heart function is part of the severe course of disease in COVID-19 infections.

In COVID-19 infections, interstitial reactive lung changes are often found in the periphery, which can then lead to consolidation and the possible consequence of pulmonary artery embolisms with peripheral infarction pneumonia. In severe disease progression, all forms of severe lung damage up to ARDS can be found. Computed tomography in high-resolution technology can visualize early interstitial changes and record them clearly [4-7, 14, 15]. 
When using dual-source CT, even with optimal contrast, pulmonary artery embolisms can be detected down to segment level $[2,5]$. For a severely ill COVID-19 patient, however, the support also means complex repositioning and transport with a high level of personnel care [19]. In addition, the contrast medium of CT imaging can pose a considerable risk to the kidneys, which are often already limited in their function. This is an advantage of contrast agent sonography (CEUS), since there is no impairment of kidney function when using sulphur hexafluoride microbbubles (SonoVue ${ }^{\circledR}$ ) $[10,11,20]$.

In preliminary studies, the potential of CEUS for a dynamic recording of organ microperfusion in severe COVID-19 infection could be demonstrated with regard to abdominal sonography [21]. However, this raises the question of accompanying changes in the CEUS dynamics from the basal lung sections near the diaphragm. However, these initial investigations indicated reactive changes in consolidation, pleural irritation with hyperemia, but also peripheral mosaic perfusion, which often occurs after peripheral microemboli. In correlation to CT, CEUS thus offers a targeted control of lung changes. However, contrast agent administration in CEUS is associated with a risk of right heart failure and arterial hypertension, but also used for early diagnosis of peripheral embolic consolidations [1, 16-18].

Considering the literature a CEUS pattern of absent or nonhomogeneous enhancement was suspicious (typical) of embolic consolidations (EC) [16-18], whereas a pattern of homogeneous PA enhancement was considered to be atypical of EC. Peripheral lesions showed a CEUS pattern suspicious of EC in $80 \%$ of the patients, with no enhancement in $40 \%$ and nonhomogeneous enhancement in another $40 \%$. A CEUS pattern of homogeneous PA enhancement, atypical of embolic consolidations, was identified in the remaining $20 \%$ of the patients. Pulmonary lesions larger than $1 \mathrm{~cm}$ showed vascularization more often than smaller lesions $(p<0.001)$ [16].

The high frame rate and high level of detail right down to the microcirculation opens up new possibilities for CEUS for targeted monitoring in the severe course of a COVID-19 infection. In particular, dynamically changing ventilation or perfusion deficits that change relatively quickly can be examined in intensive care units with appropriate US devices. The extent to which CEUS can be used to comprehensively image truly peripheral infarcts or micro embolic consolidations must be critically reviewed. At least after the confirmed diagnosis by CT, targeted monitoring can be carried out [1, $12-15,19]$.

Possible indications for the use of CEUS would be an increasing compression of basal lung sections with constriction of arterial vessels, restriction of arterial capillary microcirculation and restriction of respiratory function. In addition, CEUS also enables the dynamic assessment of organ microcirculation, especially of the liver and kidneys [21]. Here, reduced perfusion is common in severe infectious to septic clinical pictures and, as with COVID-19 patients, may require the use of ECMO treatment.

The use of CEUS for lung diagnostics is still restricted to individual cases. However, especially in the stage of increasing renal function impairment, CEUS can open up new diagnostic possibilities with regard to pulmonary changes in microvascularization. These must be examined by multicenter studies before a final evaluation is possible.

\section{References}

[1] Volpicelli G, Lamorte A, Villén T. WHAT'S NEW IN INTENSIVE CARE What's new in lung ultrasound during the COVID-19 pandemic. Intensive Care Med. 2020 https://doi.org/10.1007/s00134-020-06048-9

[2] Jędrusik P, Gaciong Z, Sklinda K, Sierpiński R, Walecki J, Gujski M. Diagnostic role of chest computed tomography in coronavirus disease 2019. Pol Arch Intern Med. 2020. doi: 10.20452/pamw.15348.

[3] $\mathrm{Hu}$ L, Wang C. Radiological role in the detection, diagnosis and monitoring for the coronavirus disease 2019 (COVID19). Eur Rev Med Pharmacol Sci. 2020;24(8):4523-4528. 
[4] Luo L, Luo Z, Jia Y, Zhou C, He J, Lyu J, Shen X. CT differential diagnosis of COVID-19 and non-COVID-19 in symptomatic suspects: a practical scoring method. BMC Pulm Med. 2020;20(1):129. doi: 10.1186/s12890-020-1170-6.

[5] Dennie C, Hague C, Lim RS, Manos D, Memauri BF, Nguyen ET, Taylor J. Canadian Association of Thoracic Radiology/Canadian Association of Radiologists Consensus Statement Regarding Chest Imaging in Suspected and Confirmed COVID-19. Can Assoc Radiol J. 2020:846537120924606.

[6] Xiang Y, Yang Q, Sun H, Qin X, Li X, Zhang Q. [Chest CT findings and their dynamic changes in patients with COVID-19]. Nan Fang Yi Ke Da Xue Xue Bao. 2020;40(3):327-332.

[7] Guo F, Zhu L, Xu H, Qin L, Liang X, Deng X. [Correlation between clinical classification of COVID-19 and imaging characteristics of MSCT volume scanning of the lungs]. Nan Fang Yi Ke Da Xue Xue Bao. 2020;40(3):321-326.

[8] Gong NM, Yin HH, Cai WH, Li QW, Wang JX, Gu CY, Wang YF, Wu J, Zhang YF. IOUS and CE-IOUS during hepatic resection for patients with hepatocellular carcinoma in liver cirrhosis. Clin Hemorheol Microcirc. 2019;71(4):483-498.

[9] Apfelbeck M, Clevert DA, Ricke J, Stief C, Schlenker B. Contrast enhanced ultrasound (CEUS) with MRI image fusion for monitoring focal therapy of prostate cancer with high intensity focused ultrasound (HIFU). Clin Hemorheol Microcirc. 2018;69(1-2):93-100.

[10] Sidhu PS, Cantisani V, Dietrich CF, Gilja OH, Saftoiu A, Bartels E, Bertolotto M, Calliada F, Clevert DA, Cosgrove D, Deganello A, D’Onofrio M, Drudi FM, Freeman S, Harvey C, Jenssen C, Jung EM, Klauser AS, Lassau N, Meloni MF, Leen E, Nicolau C, Nolsoe C, Piscaglia F, Prada F, Prosch H, Radzina M, Savelli L, Weskott HP, Wijkstra H. The EFSUMB Guidelines and Recommendations for the Clinical Practice of Contrast-Enhanced Ultrasound (CEUS) in Non-Hepatic Applications: Update 2017 (Short Version). Ultraschall Med. 2018;39(2):154-180. doi: 10.1055/s-0044101254.

[11] Sidhu PS, Cantisani V, Dietrich CF, Gilja OH, Saftoiu A, Bartels E, Bertolotto M, Calliada F, Clevert DA, Cosgrove D, Deganello A, D’Onofrio M, Drudi FM, Freeman S, Harvey C, Jenssen C, Jung EM, Klauser AS, Lassau N, Meloni MF, Leen E, Nicolau C, Nolsoe C, Piscaglia F, Prada F, Prosch H, Radzina M, Savelli L, Weskott HP, Wijkstra H. The EFSUMB Guidelines and Recommendations for the Clinical Practice of Contrast-Enhanced Ultrasound (CEUS) in Non-Hepatic Applications: Update 2017 (Long Version). Ultraschall Med. 2018;39(2):e2-e44. doi: 10.1055/a-05861107.

[12] Vetrugno L, Bove T, Orso D, Bassi F, Boero E, Ferrari G. Lung Ultrasound and the COVID-19 "Pattern": Not All That Glitters Today Is Gold Tomorrow. J Ultrasound Med. 2020. doi: 10.1002/jum.15327.

[13] Soldati G, Smargiassi A, Inchingolo R, Buonsenso D, Perrone T, Briganti DF, Perlini S, Torri E, Mariani A, Mossolani EE, Tursi F, Mento F, Demi L. On Lung Ultrasound Patterns Specificity in the Management of COVID-19 Patients. J Ultrasound Med. 2020. doi: 10.1002/jum.15326.

[14] Wan DY, Luo XY, Dong W, Zhang ZW. Current practice and potential strategy in diagnosing COVID-19. Eur Rev Med Pharmacol Sci. 2020;24(8):4548-4553.

[15] Hu L, Wang C. Radiological role in the detection, diagnosis and monitoring for the coronavirus disease 2019 (COVID19). Eur Rev Med Pharmacol Sci. 2020;24(8):4523-4528.

[16] Bartelt S, Trenker C, Görg C, Neesse A. Contrast-enhanced ultrasound of embolic consolidations in patients with pulmonary embolism: A pilot study. J Clin Ultrasound. 2016;44(3):129-35. doi: 10.1002/jcu.22313.

[17] Trenker C, Apitzsch JC, Pastor S, Bartelt S, Neesse A, Goerg C. Detection of peripheral embolic consolidations using contrast-enhanced ultrasonography in patients with no evidence of pulmonary embolism on computed tomography: A pilot study. J Clin Ultrasound. 2017;45(9):575-579. doi: 10.1002/jcu.22511.

[18] Sartori S, Postorivo S, Vece FD, Ermili F, Tassinari D, Tombesi P. Contrast-enhanced ultrasonography in peripheral lung consolidations: What's its actual role? World J Radiol. 2013;5(10):372-80.

[19] Mojoli F, Mongodi S, Orlando A, Arisi E, Pozzi M, Civardi L, Tavazzi G, Baldanti F, Bruno R, Iotti GA; COVID19 Pavia Crisis Unit. Our recommendations for acute management of COVID-19. Crit Care. 2020;24(1):207. doi: 10.1186/s13054-020-02930-6.

[20] Lamby P, Prantl L, Krüger-Genge A, Franke RP, Jung EM, Jung F. Influence of Ultrasound Microbubbles on kidney oxygen tension. Clin Hemorheol Microcirc. 2017;67(3-4):211-214.

[21] Jung EM, Stroszczinski C, Jung F. Contrast enhanced ultrasonography (CEUS)1 to detect abdominal microcirculatory2disorders in severe cases of COVID-193infection: First experience. Clin Hemorheol Microcirc. 2020. DOI:10.3233/CH-209003. 